\title{
Resuscitation in the Delivery Room: Lung Protection From the First Breath
}

\author{
Thomas E Wiswell MD
}

\author{
Introduction \\ $\mathrm{F}_{\mathrm{IO}_{2}}<1.0$ \\ CPAP and Sufficient PEEP \\ Positive-Pressure Ventilation and Sustained Inflations \\ Surfactant \\ Oropharyngeal and Intratracheal Suctioning of Meconium-Stained \\ Amniotic Fluid \\ Summary
}

Resuscitation of newborn infants occurs in approximately $10 \%$ of the more than 100 million infants born annually worldwide. The techniques used during resuscitation, such as positive-pressure ventilation and supplemental oxygen, may revive many infants, but have the potential to harm their lungs. In recent years increasing attention has been applied to providing lung protection from the first breath. This paper reviews the currently available medical evidence concerning modifying aspects of delivery room management that are thought to mitigate lung injury. These include: $\mathrm{F}_{\mathrm{IO}_{2}}<1.0$; early use of continuous positive airway pressure (CPAP) and PEEP; optimizing pressure and/or volume during ventilation; sustained inflations; need for and timing of surfactant therapy; and airway management of meconium-stained amniotic fluid. Although the evidence against $100 \%$ oxygen use is of low quality, it has been enough to alter the recommendations for oxygen use in the delivery room. It is suggested (not mandated) to use room air initially when resuscitating a termgestation infant, and to use $\mathrm{F}_{\mathrm{IO}_{2}}<1.0$ in premature infants, with $\mathrm{F}_{\mathrm{IO}_{2}}$ adjustments depending on oximetry values. Recent studies have not indicated better outcomes in premature infants in whom CPAP or PEEP is applied in the delivery room. Optimal peak ventilatory pressure and tidal volume have yet to be delineated. Although an intriguing therapy, sustained inflations have not been shown to markedly improve outcomes. Prophylactic use of surfactant in small, premature infants remains the accepted standard. Immediate placement on CPAP after surfactant instillation has yet to demonstrate clear-cut advantages. Finally, intrapartum oropharyngeal and nasopharyngeal suctioning of meconium-stained amniotic fluid does not improve outcomes in meconium-stained infants. Moreover, routine intubation and intratracheal suctioning of apparently vigorous meconiumstained infants do not improve outcomes. In summary, although multiple therapies are touted as protecting the lungs in the delivery room "from the first breath," to date there are scant supportive data. Key words: neonatal; newborn; infant; premature; resuscitation; mechanical ventilation; supplemental oxygen; $\mathrm{F}_{\mathrm{IO}_{2}}$; continuous; positive; airway; pressure; CPAP; PEEP; surfactant; meconium aspiration; sustained inflations; surfactant. [Respir Care 2011;56(9):1360-1367. (C) 2011 Daedalus Enterprises] 


\section{Introduction}

The transition from fetal to extrauterine life is complex. ${ }^{1-3}$ Although approximately $90 \%$ of infants respond well to stimulation, warmth and suctioning, the remaining $10 \%$ may need additional interventions to achieve and sustain homeostasis. Ten to twenty percent of the latter require more aggressive interventions. During the intrapartum period, the fetus and newborn must mobilize the lung fluid in the airways and parenchyma. Two to 3 days prior to delivery, the secretion of lung fluid normally declines. The labor process further reduces extravascular lung water. Subsequent to birth, fluid is driven from the alveoli into the interstitium. Many infants fail to adequately "dry out" their lungs, particularly infants delivered via cesarean section. After parturition, the airways and the alveoli have to be inflated to exchange gas. Poor respiratory effort impedes this process. Preterm infants with insufficient surfactant will have widespread atelectasis. Small preterm infants and depressed or neurologically impaired termgestation infants may not be able to generate sufficient negative intrathoracic pressure to expand their airways. Pulmonary vascular resistance has to decline to allow a 10 -fold increased flow of blood to the lungs. This decrease is modulated by increased oxygenation and adequate ventilation. Additionally, peripheral vascular resistance has to increase to increase systemic arterial blood pressure, which helps drive blood into the lungs, allowing the exchange of oxygen and carbon dioxide. The intrauterine fetal circulation changes to adapt to extrauterine life. In utero, the ductus arteriosus and foramen ovale serve as conduits of oxygenated blood from the right side of the heart to the systemic circulation. These pathways must functionally and then physiologically close once the newborn is cut off from placental flow with clamping of the umbilical cord. Failure of the ductus arteriosus and foramen ovale to adjust can result in shunting of the blood and adversely affect gas exchange.

Premature infants have particular difficulties in accomplishing normal transition. ${ }^{4}$ Their lungs are structurally

Dr Wiswell is affiliated with the Center for Neonatal Care and the Department of Neonatology, Florida Hospital for Children, Orlando, Florida.

Dr Wiswell presented a version of this paper at the 47th RESPIRATORY CARE Journal Conference, "Neonatal and Pediatric Respiratory Care: What Does the Future Hold?" held November 5-7, 2010, in Scottsdale, Arizona.

The author has disclosed no conflicts of interest.

Correspondence: Thomas E Wiswell MD, Center for Neonatal Care, 2718 North Orange Avenue, Suite B, Orlando FL 32804. E-mail: thomas_wiswell@yahoo.com.

DOI: $10.4187 /$ respcare. 01433 immature and frequently deficient in surfactant. They are less able to get rid of the copious intraluminal lung fluid present in utero. The lack of a stiff chest wall contributes to widespread atelectasis. Inflammation is frequently present in the lungs of these infants, compounding the effects of increased pulmonary vascular resistance and causing surfactant dysfunction. The majority of preterm infants less than 30 weeks gestation require extra assistance in the delivery room.

Typical interventions used to assist the neonate's transition are supplemental oxygen and continuous positive airway pressure (CPAP) or active ventilation, via face mask or endotracheal tube. Premature infants often receive exogenous surfactant in the minutes following birth. Most of the time these therapies work well, and the infant can sustain his or her own respirations and gas exchange within minutes. Nonetheless, these tools have the potential to adversely affect the lungs, the very organ that clinicians, with the best of intentions, have been trying to help.

Based on animal and human research, a number of mechanisms have been described that could injure lungs during resuscitation. Volutrauma refers to overdistention of areas of the lung that can occur with assisted ventilation. Inflammation and physical damage may be consequences. Even a few large manual breaths can cause injury. ${ }^{5}$ Atelectrauma is injury due to repeated collapse and reopening of alveoli and the distal bronchioles. The air sacs are exposed to repeated shearing forces, with inflammation, membrane disruption, and surfactant dysfunction. The main mechanism producing atelectrauma is believed to be the use of inadequate PEEP, resulting in low residual volume and atelectasis. Biotrauma refers to injurious local and systemic inflammatory responses to physical stress. Finally, high $\mathrm{F}_{\mathrm{IO}_{2}}$ could be toxic to the lungs. Oxygen free radicals may be formed and inflammation may occur. Neonates have less antioxidant capability than older children.

Historically, during resuscitation $100 \%$ oxygen has been used while ventilating neonates. ${ }^{6}$ There has been recent recognition that this practice could have adverse consequences. Finally, meconium-stained amniotic fluid is found in approximately $12-15 \%$ of all births, and meconium may be aspirated either in utero or postnatally. Meconium can have direct toxic effects on the lung parenchyma itself, and the meconium aspiration syndrome may result in a need for substantial respiratory support, which could have deleterious effects on the lung. ${ }^{7}$

A number of lung-protective strategies during newborn resuscitation have been proposed, which I will discuss in the body of this paper.

$$
\mathrm{F}_{\mathrm{IO}_{2}}<1.0
$$

One of the goals of resuscitation is to provide sufficient oxygen perfusion of tissue. Oxygen is critical for life and 
is required for cell metabolism and growth. Even relatively short-term hypoxemia can have disastrous outcomes. Oxygen was first administered to a neonate in 1780. Since the 1940s, oxygen has been the most commonly used therapy in the delivery room and in the neonatal intensive care unit. ${ }^{8}$ High $\mathrm{F}_{\mathrm{IO}_{2}}$ for a prolonged period (days to weeks) is a major factor in the pathogenesis of major newborn morbidity, including retinopathy of prematurity and bronchopulmonary dysplasia (BPD). High $\mathrm{F}_{\mathrm{IO}_{2}}$ leads to the production of oxygen free radicals such as superoxide, hydrogen peroxide, and the hydroxyl radical. These are highly reactive chemical species that can react with and injure almost every type of molecule in living cells. High $\mathrm{F}_{\mathrm{IO}_{2}}$ may prolong the time to spontaneous crying and breathing, increase oxygen consumption, decrease minute ventilation, cause atelectasis, or alter cerebral circulation (decrease cerebral blood flow). ${ }^{9-17}$ By contrast, too low an $\mathrm{F}_{\mathrm{IO}_{2}}$ can cause persistent pulmonary hypertension of the newborn, hypoxic brain injury, multisystem organ dysfunction, and is more likely to keep the ductus arteriosus open.

Several trials have assessed the initial use of room air versus $100 \%$ oxygen in the resuscitation of newborns. ${ }^{9-14}$ Although the bulk of enrolled patients were of term gestation, infants as small as $1,000 \mathrm{~g}$ birth weight and 27 weeks gestation were included in 4 of the trials. ${ }^{9-11,14}$ In general, these studies indicated that using room air as a starting point during resuscitation resulted in similar outcomes to $100 \%$ oxygen. Most of the investigations found that infants resuscitated with room air took less time to have the onset of both their first breath and their first cry. Approximately one quarter of the room air-treated neonates needed to be switched to $100 \%$ oxygen after several minutes because of a failure to respond. There have been many criticisms of these investigations. The 4 trials done in developing countries ${ }^{9-11,14}$ were quasi-randomized, they enrolled patients into the treatment groups depending on whether it was an odd-number versus even-number day, and the investigators were not blinded to the $\mathrm{F}_{\mathrm{IO}_{2}}$ children received. Two of the trials ${ }^{10,12}$ found trends toward higher mortality in the $100 \%$ oxygen groups. The other 4 studies, including the 2 performed in a developed country, ${ }^{12,13}$ did not find a similar trend. Two trials revealed increases in markers of oxidative stress (eg, serum levels of glutathione and superoxide dismutase) among those treated with $100 \%$ oxygen. Short-term neurological outcomes (assessed at 3-28 days) were reportedly equivalent in all 6 investigations. The 4 studies that enrolled preterm infants did not stratify outcomes by gestational age.

Sorely lacking in all of these reports were outcome data concerning major morbidity: chronic lung disease, persistent pulmonary hypertension, intracranial hemorrhage, cerebral white matter injury, necrotizing enterocolitis, patent ductus arteriosus, and retinopathy of prematurity. There
Table 1. Target Preductal $\mathrm{S}_{\mathrm{pO}_{2}}$ Ranges After Birth*

\begin{tabular}{cc}
\hline \hline Time After & $\begin{array}{c}\text { Desired Preductal } \\
\text { Oxygen Saturation } \\
\text { Birth (min) }\end{array}$ \\
\hline 1 & $60-65$ \\
2 & $65-70$ \\
3 & $70-75$ \\
4 & $75-80$ \\
5 & $80-85$ \\
6 & $85-95$
\end{tabular}

* If a neonate undergoing resuscitation has preductal oxygen saturation below the recommended level, blended oxygen should be administered in sufficient concentration to achieve the desired oxygen saturation. (Adapted from Reference 19.)

was only one long-term follow-up study, of the infants in the largest trial..$^{15}$ Approximately one third of infants in that trial were subsequently assessed in an unblinded fashion at 18-24 months following enrollment. The examiners were investigators from the original study and were not neurologists. They administered a questionnaire and examined the children. Cognition was not assessed. There were no differences in the outcomes between the groups in that limited follow-up.

The data generated by these studies have been controversial. Interestingly, a group of independent authors, using the aforementioned data, published meta-analyses in 2 separate reports published within months of each other and drew opposite conclusions. ${ }^{16,17}$ In their initial publication, a Cochrane Systematic Review, ${ }^{16}$ they concluded that the evidence was insufficient to recommend room air over $100 \%$ oxygen. Several months later, using the same data and analytical methods, they concluded that room air should initially be used for resuscitating term and near-term infants, with oxygen as a backup. ${ }^{17}$ Despite the limitations of the 6 investigations, the International Liaison Committee on Resuscitation recently revised its recommendations concerning the use of oxygen: "In term infants receiving resuscitation at birth with positive-pressure ventilation, it is best to begin with air rather than $100 \%$ oxygen. If, despite effective ventilation, there is no increase in heart rate, or if oxygenation (guided by oximetry) remains unacceptable, use of a higher concentration of oxygen should be considered." 18 This was stated to be a class IIb, level of evidence $\mathrm{B}$ recommendation, ${ }^{19}$ meaning that it is an acceptable recommendation based on "weak" evidence (not excellent or good evidence). In the recently published 6th edition of the Textbook of Neonatal Resuscitation, published under the auspices of The Neonatal Resuscitation Program, appears a table of preductal saturation target ranges during resuscitation (Table 1). ${ }^{19,20}$ If an infant's oxygen saturation is below the lower limit at specified time points, it is recommended that oxygen should be administered, via blender, in concentration sufficient to achieve the target range, and 
that use of room air is advocated (but not mandated) at the initiation of resuscitation in a term infant. ${ }^{20}$

What should one do concerning supplemental oxygen when resuscitating a premature infant? In the 4 aforementioned studies, the premature infants' outcomes were not delineated. The optimal oxygen saturation for the resuscitation, stabilization, and ongoing care of the very preterm infant remains undefined. ${ }^{21}$ There have been several small, randomized controlled trials of lower versus higher $\mathrm{F}_{\mathrm{IO}_{2}}$ during neonatal resuscitation of preterm infants. ${ }^{22-27}$ Only one of the trials used room air as their initial low- $\mathrm{F}_{\mathrm{IO}_{2}}$ group, ${ }^{22}$ and every one of those infants needed supplemental oxygen within $2-5 \mathrm{~min}$ of birth. Similar to the termgestation infant trials, there appeared to be fewer markers of oxidative stress among infants treated with lower $\mathrm{F}_{\mathrm{IO}_{2}}{ }^{23,25,27}$ None of those trials were adequately powered to evaluate important long-term outcomes such as survival, neurodevelopmental disability, or BPD. For premature infants the International Liaison Committee on Resuscitation recommends: "Because many preterm infants of $<32$ weeks gestation will not reach target saturation on room air, blended oxygen and air may be given judiciously, and is ideally guided by pulse oximetry. Both hyperoxemia and hypoxemia should be avoided." 18 The new 6th edition of the Textbook of Neonatal Resuscitation advocates "something less than $100 \%$ oxygen, but more than $21 \%$ oxygen for a preterm baby." 20 In my unit, when resuscitating a premature infant we start with an initial $\mathrm{F}_{\mathrm{IO}_{2}}$ of 0.50 and adjust upward or downward depending on the preductal oxygen saturation during the resuscitation process. A large well designed randomized controlled blinded prospective trial is needed to determine the appropriate $\mathrm{F}_{\mathrm{IO}_{2}}$ when initiating resuscitation.

In his classic tome Retrolental Fibroplasia: A Modern Parable, ${ }^{28}$ the late William Silverman, the "grandfather" of randomized controlled trials in neonates, related a story of oxygen restriction in preterm infants. During the 1950s it was recognized that premature infants given high $\mathrm{F}_{\mathrm{IO}_{2}}$ during hospitalization were more likely to develop the serious, potentially blinding condition of retrolental fibroplasia (ie, retinopathy of prematurity). There was subsequent widespread restriction of oxygen use in premature infants. Only after the ensuing 5 years was it recognized that this oxygen restriction had the unintended consequence of increasing mortality and cerebral palsy in survivors. Additionally, several large trials are underway to compare 2 different target oxygen saturation ranges (85-89\% and $91-95 \%$ ) in premature infants $<28$ weeks gestation, and Stenson ${ }^{29}$ recently examined mortality data from more than 3,600 infants in those trials. He found a significant $8.5 \%$ higher frequency of death among infants in the lower target saturation range, and the difference appeared to be mainly due to more deaths from brain injury and necrotizing enterocolitis. I would caution clinicians to be judi- cious about jumping on the low- $\mathrm{F}_{\mathrm{IO}_{2}}$ or room air bandwagon.

\section{CPAP and Sufficient PEEP}

The lungs of preterm infants at birth are surfactant deficient, so it may be difficult for them to achieve adequate functional residual capacity (FRC) and maintain open alveoli and distal airways. The delivery room use of CPAP or PEEP of at least $4-5 \mathrm{~cm} \mathrm{H}_{2} \mathrm{O}$ has been advocated to assist in lung expansion, establish FRC, and improve oxygenation. ${ }^{1,3,4}$ Early use of CPAP in the delivery room, in both preterm and term-gestation infants, has been enthusiastically advocated by many clinicians, ${ }^{20}$ but there is a scarcity of data supporting this approach.

Finer et $\mathrm{al}^{30}$ assessed the effects of CPAP or PEEP in infants $<28$ weeks gestation. One-hundred four infants were randomized to either no CPAP and $100 \%$ oxygen or to CPAP/PEEP via a T-piece resuscitator (NeoPuff, Fisher \& Paykel, Auckland, New Zealand). Delivery room intubation was needed in $41 \%$ of the controls and $49 \%$ of the CPAP/PEEP group, whereas there was no significant difference in intubation at some other time during hospitalization ( $78 \%$ vs $82 \%$ ). Death was more likely in the CPAP/ PEEP group (27\% vs $13 \%, P=.07$ ), as was the incidence of pneumothoraces $(13 \%$ vs $9 \%$, difference not significant). The frequency of chronic lung disease was not assessed.

Morley and colleagues ${ }^{31}$ randomized more than 600 premature infants (25-28 weeks gestation) in the delivery room to CPAP $\left(8 \mathrm{~cm} \mathrm{H}_{2} \mathrm{O}\right)$ or to intubation and ventilation. Although the CPAP group had a trend toward a lower combined end point of death or BPD (34\% vs 39\%), the difference was not significant. In the CPAP group there was less use of surfactant, and the subjects averaged one day less of mechanical ventilation. Unfortunately, the CPAP-treated infants were significantly more likely to develop pneumothoraces $(9 \%$ vs $3 \%, P<.001)$.

The SUPPORT trial ${ }^{32}$ involved infants born between 24 and 27 weeks gestation. The infants were randomized to either intubation and surfactant within 1 hour of birth, or to CPAP $\left(5 \mathrm{~cm} \mathrm{H}_{2} \mathrm{O}\right)$ initiated in the delivery room. The CPAP patients could subsequently be intubated and receive surfactant if they fulfilled certain criteria $\left(\mathrm{F}_{\mathrm{IO}_{2}}>0.50\right.$, $\mathrm{P}_{\mathrm{aCO}_{2}}>65 \mathrm{~mm} \mathrm{Hg}$, or hemodynamic instability). There was a combined primary outcome: death or BPD. Eightythree percent of the CPAP group subsequently required intubation. The CPAP group had approximately 3 fewer days of mechanical ventilation. Nevertheless, there were no statistically significant differences in death, BPD, or the combined end point of death or BPD.

Members of the Vermont Oxford Network ${ }^{33}$ performed a prospective randomized controlled trial of 3 approaches in the delivery room. Preterm infants $(n=648)$ of $26-$ 
29 weeks gestation were randomized to either: prophylactic surfactant followed by ventilation; intubation and surfactant followed by rapid extubation to nasal CPAP; or early stabilization on nasal CPAP. The nasal CPAP group was less likely to receive surfactant or to be ventilated during the first week of life. However, there were no differences between groups in the outcomes of death, chronic lung disease, or other complications of prematurity.

Finally, the Brazilian Network on Neonatal Research ${ }^{34}$ also assessed early use of nasal CPAP in the delivery room. They randomized 173 infants (birth weight 1,000$1,499 \mathrm{~g})$ to either nasal CPAP $\left(5 \mathrm{~cm} \mathrm{H}_{2} \mathrm{O}\right)$ immediately in the delivery room, or to nasal CPAP subsequently when clinically indicated. Early use of nasal CPAP in the delivery room did not improve the frequency of surfactant use, ventilator use, pneumothoraces, or BPD.

I would conclude from the aforementioned trials that the current evidence to support routine use of CPAP/PEEP in the delivery room among preterm infants is less than compelling. If we examine the major end points of death or $\mathrm{BPD}$, the use of CPAP/PEEP in the delivery room does not influence outcomes. Finally, there are virtually no scientific data on the delivery room use of CPAP or PEEP in term-gestation infants. The International Liaison Committee on Resuscitation report ${ }^{18}$ and the Neonatal Resuscitation Program guidelines ${ }^{20}$ suggest that the use of CPAP/ PEEP should reflect local expertise and preferences.

\section{Positive-Pressure Ventilation and Sustained Inflations}

The depressed infant frequently requires positive-pressure ventilation. In an animal model, ${ }^{5}$ as few as 6 large manual breaths $(35-40 \mathrm{~mL} / \mathrm{kg}$ ) are enough to cause widespread histologic lung injury. Unfortunately, there is no single appropriate tidal volume or peak inspiratory pressure that is appropriate for all infants, as lung compliance varies from infant to infant. Additionally, spontaneously breathing infants may initially generate extraordinarily high negative intrathoracic pressure (up to $70 \mathrm{~cm} \mathrm{H}_{2} \mathrm{O}$ ) in an effort to achieve FRC. ${ }^{2}$ No trials have compared the effect on outcomes of different inflation pressures or tidal volumes during initial stabilization of preterm infants. ${ }^{35}$ In general, the initial peak inflating pressure used, in both premature and term infants, should be individualized to achieve an increase in heart rate or movement of the chest. Higher inflation pressure is typically needed for the initial few breaths to open the lungs, but the clinician should be cognizant that with subsequent breaths lower peak inflating pressure or tidal volume is probably required. There are virtually no data on pulmonary mechanics (eg, compliance, resistance, pressure-volume relationships) during resuscitation of neonates. In term infants an initial inflating pressure of approximately $30 \mathrm{~cm} \mathrm{H}_{2} \mathrm{O}$ is sufficient to improve heart rate or chest expansion, whereas in preterm infants $20-25 \mathrm{~cm} \mathrm{H}_{2} \mathrm{O}$ is generally effective. ${ }^{18}$ There have been no scientific inves- tigations of different tidal volumes during or immediately after resuscitation. Clearly, clinical trials are needed to assess various strategies in establishing lung volume, FRC, shortterm outcomes (eg, oxygenation, ventilation, spontaneous breathing), and long-term outcomes (eg, mortality, chronic lung disease, other markers of lung injury).

Sustained inflations for the first several breaths of manual ventilation are a popular method to assist the formation of FRC during resuscitation. ${ }^{35}$ This concept dates back 3 decades. ${ }^{36}$ Most work has been done in the extremelylow-birth-weight population. Linder and colleagues ${ }^{37}$ used sustained inflations of $20-25 \mathrm{~cm} \mathrm{H}_{2} \mathrm{O}$ for 15-20 seconds, followed by CPAP, and compared the outcomes to historical controls. The infants were less likely to require intubation, either in the delivery room or thereafter. However, when Linder et al ${ }^{38}$ subsequently performed a randomized controlled trial to compare their sustained-inflations strategy to routine intubation/ventilation, they found no differences in the number of infants subsequently requiring intubation or mechanical ventilation.

Te Pas and Walther ${ }^{39}$ randomized 207 preterm infants who required resuscitation to either a sustained inflation of $20 \mathrm{~cm} \mathrm{H}_{2} \mathrm{O}$ for 10 seconds or to conventional positivepressure ventilation with a self-inflating bag and mask with an initial inflation pressure of $30-40 \mathrm{~cm} \mathrm{H}_{2} \mathrm{O}$. The sustained-inflation group was significantly less likely to require intubation within the first 72 hours of life (37\% vs $51 \%$ ), had a shorter duration of ventilation, and were less likely to develop BPD (22\% vs $34 \%$ ).

Lista and colleagues ${ }^{40}$ compared a group of premature infants treated with sustained inflations $\left(25 \mathrm{~cm} \mathrm{H}_{2} \mathrm{O}\right.$ for $15 \mathrm{~s})$ to a historical cohort treated with $\mathrm{CPAP}$ at $5 \mathrm{~cm} \mathrm{H}_{2} \mathrm{O}$. The sustained-inflations group was less likely to require mechanical ventilation or to develop BPD. However, the fact that the study was not prospective, randomized, or controlled limits its usefulness. Over the past 30 years there have been virtually no investigations, in term-gestation infants, of sustained inflations versus other respiratory management techniques. Therefore, although sustained inflations appear to be an intriguing method of opening the lung and achieving an initial FRC, I do not believe there are currently enough data to support the routine use of sustained inflations during resuscitation of preterm or termgestation infants. The International Liaison Committee on Resuscitation report ${ }^{18}$ and the Neonatal Resuscitation Program guideline ${ }^{20}$ suggest that decisions regarding variable inflation times, such as sustained inflations, should reflect local expertise and preferences.

\section{Surfactant}

Exogenous surfactant therapy has been an integral part of neonatal intensive care unit care for the past 20 years. In the preterm infant population, surfactant use has sub- 
stantially decreased mortality. However, it has not affected BPD incidence. Premature lung disease (respiratory distress syndrome) results from surfactant deficiency in a structurally immature lung that is easily injured. ${ }^{41}$ The major surfactants used throughout the world in 2011 are animal-derived, generally from a mince or lavage of either bovine or porcine lungs. ${ }^{42}$

Outside the preterm population, there are several disorders in which surfactant deficiency or inactivation occurs (eg, meconium aspiration syndrome, pneumonia). Several trials have found exogenous surfactant effective in nonpremature infants. In a systematic review, Soll and colleagues ${ }^{43}$ concluded that prophylactic use (generally defined as within the first $15 \mathrm{~min}$ of life) of exogenous surfactant in premature infants is better than selective (rescue) use of surfactant among infants $<29$ weeks gestation (fewer pneumothoraces, decreased mortality, less pulmonary interstitial emphysema). Prophylaxis is generally done in the delivery room soon after birth. Most of the data supporting the superiority of prophylaxis come from studies performed in the late 1980s and early 1990s, which was before the widespread use of antenatal corticosteroids (to hasten lung maturation) and newer methods of support (eg, high-frequency ventilation) and modes of conventional ventilation (eg, synchronization, volume-targeting, pressure support). Additionally, many believe that CPAP at a sufficient level accomplishes results similar to exogenous surfactant (by opening the alveoli and improving oxygenation). Thus, frequently clinicians are now delaying surfactant administration until they are certain respiratory distress syndrome is established and is more than mildly debilitating.

The surfactant approach used in the Intubation, Surfactant, Extubation to CPAP (INSURE) trial has been widely practiced in Europe over the past decade ${ }^{44}$ and has gained popularity in other areas of the world. Although most randomized controlled trials of the INSURE approach have found a short-term decrease in the need for mechanical ventilation, no differences in mortality or chronic lung disease have been described. ${ }^{44}$ The Vermont Oxford Network trial ${ }^{33}$ found no advantages with the INSURE approach. The current place of INSURE therapy during resuscitation needs to be clarified.

Do we need new surfactants? With currently available animal-derived surfactants, up to $15 \%$ of premature infants fail to respond. In the commercially available surfactants there is wide variability $( \pm 50 \%)$ of target surfactant protein level, and potential immunogenicity and infection risks. Synthetic surfactant that contains surfactant protein mimics may be one way of improving our current therapies. ${ }^{42}$ In addition, noninvasive delivery (eg, aerosolization or via laryngeal mask airway) may prove useful in certain situations.
Although surfactant therapy is used daily in delivery rooms around the world in premature infants, to date neither the International Liaison Committee on Resuscitation nor the Neonatal Resuscitation Program have addressed surfactant use in the delivery room. Thus, clinicians have to make their own decisions on the timing of surfactant therapy in the vulnerable population of preterm infants.

\section{Oropharyngeal and Intratracheal Suctioning of Meconium-Stained Amniotic Fluid}

Prevention of meconium aspiration syndrome ${ }^{7}$ has long been a goal of clinicians in the delivery room. Approximately $12-15 \%$ of all newborn infants are born through meconium-stained amniotic fluid, and, historically, 3-10\% of those patients develop meconium aspiration syndrome. ${ }^{7,45,46}$ It is associated with substantial morbidity, such as need for mechanical ventilation, persistent pulmonary hypertension of the newborn, need for extracorporeal membrane oxygenation, long-term abnormal pulmonary dysfunction, and death. Histological features of meconium aspiration syndrome include inflammation, obstruction of airways, and areas of atelectasis alternating with overexpansion. Meconium can inactivate endogenous surfactant and can cause direct injury to the lung parenchyma, including necrosis and hemorrhage. Multiple maneuvers have been used in the delivery room to prevent meconium aspiration $7,45,46$ The hope has been that removal of meconium-stained amniotic fluid would mitigate its effects on the infant's lungs.

For a 25 -year period starting in the mid-1970s, the routine teaching was to intubate all meconium-stained infants and suction out the trachea. ${ }^{7}$ We performed a large randomized controlled trial to assess whether this maneuver would be effective in apparently vigorous meconiumstained neonates. ${ }^{45} \mathrm{We}$ enrolled more than 2,000 infants. If they were thought to be vigorous immediately after delivery (heart rate $>100$ beats/min, good respiratory effort, normal tone), they were randomized to either intubation and tracheal suctioning or to expectant management. We found no differences in outcomes between the 2 groups. Additionally, since the mid-1970s it was routine when delivering a meconium-stained infant to suction out the nasopharynx and oropharynx before the head and shoulders are delivered. ${ }^{7}$ The thinking was that removing the meconium-stained fluid before the child's first breath would prevent aspiration. We performed a large, randomized controlled trial to assess whether this therapy was truly effective. ${ }^{46} \mathrm{We}$ enrolled more than 2,000 meconium-stained infants who were randomized to either intrapartum oropharyngeal and nasopharyngeal suctioning or to expectant management. There were no differences in outcomes between the 2 groups. These results have contributed to the thinking that meconium aspiration syndrome is often a 
result of in utero aspiration of meconium-stained amniotic fluid and cannot be prevented by intrapartum or postnatal therapies. There remains a major question of whether the depressed meconium-stained infant benefits from intratracheal suctioning, as has long been recommended by the International Liaison Committee on Resuscitation and the Neonatal Resuscitation Program. Over the past decade there have been many discussions about performing a definitive clinical trial, but at present there are no trials underway to answer this question.

\section{Summary}

Newly born infants frequently need assistance to establish normal respirations, but many resuscitation maneuvers can injure the lungs of preterm and term-gestation neonates. I have discussed the rationale for preventing such injury and the evidence basis for what we do. To date, however, there are no good scientific data supporting changes in our current delivery room management of neonates that can clearly increase lung protection and improve overall outcomes.

\section{REFERENCES}

1. Bry K. Newborn resuscitation and the lung. NeoReviews 2008;9: e506-e511.

2. Tzong-Jin W, Warlo WA. Pulmonary physiology of neonatal resuscitation. NeoReviews 2001;2:e45-e50.

3. Leone TA, Finer NN. Neonatal resuscitation: beyond the basics. NeoReviews 2005;6:e177-e183.

4. Schmölzer GM, Te Pas AB, Davis PG, Morley CJ. Reducing lung injury during neonatal resuscitation of preterm infants. J Pediatr 2008;153(6):741-745.

5. Björklund LJ, Ingimarsson J, Curstedt T, John J, Robertson B, Werner O, Vilstrup CT. Manual ventilation with a few large breaths at birth compromises the therapeutic effect of subsequent surfactant replacement in immature lambs. Pediatr Res 1997;42(3):348-355.

6. Rabi Y. Oxygen and resuscitation of the preterm infant. NeoReviews 2010;11:e130-e137.

7. Wiswell TE. Handling of the meconium-stained infant. Semin Neonatol 2001;6(3):225-231.

8. Zaichkin J, Wiswell TE. The history of neonatal resuscitation. Neonatal Netw 2002;21(5):21-28.

9. Ramji S, Ahuja S, Thirupuram S, Rootwelt T, Rooth G, Saugstad OD. Resuscitation of asphyxic newborn infants with room air or 100\% oxygen. Pediatr Res 1993;34(6):809-812.

10. Saugstad OD, Rootwelt T, Aalen O. Resuscittiton of asphyxiated newborn infants with room air or oxygen: an international controlled trial: the Resair 2 Study. Pediatrics 1998;102(1)e1.

11. Ramji S, Rasaily R, Mishra PK, Narang A, Jayam S; Kapoor AN, et al. Resuscitation of asphyxiated newborns with room air or $100 \%$ oxygen at birth: a multicentric clinical trial. Indian Pediatrics 2003; 40:510-517.

12. Vento M, Asensi M, Sastre J, Garcia-Sala F, Pallardo FV, Viña J. Resuscitation with room air instead of $100 \%$ oxygen prevents oxidative stress in moderately asphyxiated term neonates. Pediatrics 2001;107(4):642-647.
13. Vento M, Asensi M, Sastre J, Lloret A, Garcia-Sala F, Viña J. Oxidative stress in asphyxiated term infants resuscitated with $100 \%$ oxygen. J Pediatr 2003;142(3):240-246. Erratum in: J Pediatr 2003; 142(6):616.

14. Bajaj N, Udani RH, Nanavati RN. Room air vs. 100 per cent oxygen for neonatal resuscitation: a controlled clinical trial. J Trop Pediatr 2005;51(4):206-211.

15. Saugstad OD, Ramji S, Irani SF, El-Meneza S, Hernandez EA, Vento M, et al. Resuscitation of newborn infants with $21 \%$ or $100 \%$ oxygen: follow-up at 18 to 24 months. Pediatrics 2003;112(2):296-300.

16. Tan A, Schulze A, O'Donnell CP, Davis PG. Air versus oxygen for resuscitation of infants at birth. Cochrane Database Syst Rev 2004(3): CD002273.

17. Davis PG, Tan A, O'Donnell CP, Schulze A. Resuscitation of newborn infants with $100 \%$ oxygen or air: a systematic review and meta-analysis. Lancet 2004;364(9442):1329-1333.

18. Perlman JM, Wyllie J, Kattwinkel J, Atkins DL, Chameides L, Goldsmith JP, et al. Neonatal resuscitation 2010 international consensus on cardiopulmonary resuscitation and emergency cardiovascular care science with treatment recommendations. Circulation 2010;122(Suppl 2):S516-S538.

19. Kattwinkel J, Perlman JM, Aziz K, Colby C, Fairchild K, Gallagher J, et al. Neonatal resuscitation: 2010 American Heart Association guidelines for cardiopulmonary resuscitation and emergency cardiovascular care. Pediatrics 2010;126(5):e1400-e1413.

20. Kattwinkel J, and the AHA/AAP Neonatal Resuscitation Program Steering Committee, editors. Textbook of neonatal resuscitation, 6th edition. Elk Grove Village, IL: American Academy of Pediatrics; 2011.

21. Finer NN, Saugstad O, Vento M, Barrington K, Davis P, Duara S, et al. Use of oxygen for resuscitation of the extremely low birth weight infant. Pediatrics 2010;125(Suppl):389-391.

22. Wang CL, Anderson C, Leone TA, Rich W, Govindaswami B, Finer NN. Resuscitation of preterm neonates by using room air or $100 \%$ oxygen. Pediatrics 2008;121(6):1083-1089.

23. Escrig R, Arruza L, Izquierdo I, Villar G, Saenz P, Gimeno A, Moro M, Vento M. Pediatrics 2008;121(5):875-881.

24. Rabi Y, Nettel-Aguirre A; Singhal N. Room air versus oxygen administration during resuscitation of preterm infants (ROAR study). E-PAS 2008;5127-5135.

25. Vento M, Moro M, Escrig R, Arruza L, Villar G, Iszuierdo I, et al. Preterm resuscitation with low oxygen causes less oxidative stress, inflammation, and chronic lung disease. Pediatrics 2009;124(3):e439e449.

26. Harling AE, Beresford MW, Vince GS, Bates M, Yoxall CW. Does use of 50\% oxygen at birth in preterm infants reduce lung injury? Arch Dis Child Fetal Neonatal Ed 2005;90(5):F401-F405.

27. Ezaki S, Suzuki K, Kurishima C, Miura M, Weilin W, Hoshi R, et al. Resuscitation of preterm infants with reduced oxygen results in less oxidative stress than resuscitation with $100 \%$ oxygen. J Clin Biochem Nutr 2009;44(1):111-118.

28. Silverman WA. Retrolental fibroplasia: a modern parable. New York: Grune \& Stratton; 1980.

29. Stenson BJ. Interim safety meta-analysis of survival at 36 weeks gestation in studies contribution to the NeOProM oxygen saturation targeting trials collaboration. Pediatric Academic Societies conference 2011. Abstract 3123.4.

30. Finer NN, Carlo WA, Duara S, Fanaroff AA, Donovan EF, Wright LL, et al. Delivery room continuous positive airway pressure/positive end-expiratory pressure in extremely low birth weight infants: a feasibility trial. Pediatrics 2004;114(3):651-657.

31. Morley CJ, Davis PG, Doyle LW, Brion LP, Hascoet JM, Carline JB; and the COIN Trial Investigators. Nasal CPAP or intubation at birth 
for very preterm infants. N Engl J Med 2008;358(7):700-708. Erratum in: N Engl J Med 2008;358(14):1529.

32. Finer NN, Carlo WA, Walsh MC, Rich W, Gantz MG, Laptook AR, et al; and the SUPPORT Study Group of the Eunice Kennedy Shriver NICHD Neonatal Research Network. Early CPAP versus sufactant in extremely preterm infants. N Engl J Med 2010;362(21):1970-1979.

33. Dunn M. Delivery room management of preterm infants at risk for respiratory distress syndrome (RDS). Pediatric Academic Societies conference 2010. Abstract 1670.2.

34. Goncalves WA. CPAP application to very low birth weight infants in the delivery room: a randomized clinical trial. Pediatric Academic Societies conference 2010. Abstract 1472.219.

35. O’Donnell CPF, Stenson BJ. Respiratory strategies for preterm infants at birth. Semin Fetal Neonatal Med 2006;13:401-409.

36. Vyas H, Milner AD, Hopkin IE, Boon AW. Physiologic responses to prolonged and slow-rise inflation in the resuscitation of the asphyxiated newborn infant. J Pediatr 1981;99(4):635-639.

37. Lindner W, Voss beck S, Hummler H, Phllandt F. Delivery room management of extremely low birth weight infants: spontaneous breathing or intubation? Pediatrics 1999;40(2):71-88.

38. Linder W, Hogel J, Pohlandt. Sustained pressure-controlled inflation or intermittent mandatory ventilation in preterm infants in the delivery room? A randomized, controlled trial on initial respiratory support via nasopharyngeal tube. Acta Paediatr 2005;94(3):303-309.
39. te Pas AB, Walther FJ. A randomized, controlled trial of deliveryroom respiratory management in very preterm infants. Pediatrics 2007;120(2):322-329. Erratum in: Pediatrics 2007;120(4):936.

40. Lista G, Fontana P, Castoldi F, Cavigioli F, Dani C. Does sustained lung inflation at birth improve outcome of preterm infants at risk for respiratory distress syndrome? Neonatology 2011;99(1):45-50.

41. Jobe AH. Why surfactant works for respiratory distress syndrome. NeoReviews 2006;7:e95-e105.

42. Wiswell TE. Extended use of surfactant therapy. Clin Perinatol 2001; 28(3):695-711.

43. Soll RF, Morley CJ. Prophylactic versus selective use of surfactant in preventing morbidity and mortality in preterm infants. Cochrane Database Syst Rev 2000;(2):CD000510.

44. Wiswell TE, Courtney SE. Noninvasive respiratory support. In: Goldsmith JP, Karotkin EH, editors. Assisted ventilation of the neonate, 5th edition. St. Louis: Elsevier; 2011:140-162.

45. Wiswell TE, Gannon CM, Jacob J, Goldsmith L, Szyld E, Weiss K, et al. Delivery room management of the apparently vigorous meconium-stained neonate: results of the multicenter, international collaborative trial. Pediatrics 2000;105(1 Pt 1):1-7.

46. Wiswell TE, Knight GR, Finer NN, Donn SM, Desai H, Walsh WF, et al. A multicenter, randomized, controlled trial comparing Surfaxin (lucinactant) lavage with standard care for treatment of the meconium aspiration syndrom. Pediatrics 2002;109(6):1081-1087.

\section{Discussion}

Walsh: It's my understanding that neonates lack antioxidants, and you talked about the negative impact of free radicals. Are there any studies that support the use of antioxidants in the delivery room?

Wiswell: None have been specifically used in the delivery room. For the antioxidants used later in neonates there is little evidence that they're effective for any lung disorders such as respiratory distress syndrome or BPD. Have any of you used superoxide dismutase (SOD), administered down the endotracheal tube? I was involved with some of the initial work in the 1990s. As an aside, our therapists said that "SOD" must stand for "sudden onset of death," because frequently the babies who received it desaturated and became bradycardic.

Walsh: With the approval of the use of $\mathrm{F}_{\mathrm{IO}_{2}}<1.0$ in the delivery room, do you think we'll use more recruitment maneuvers for better management of FRC, to keep oxygen saturation up?
Wiswell: Hopefully, the latter. I'm a firm believer in achieving FRC but not overdoing it. Once you've achieved good FRC with an optimal mean airway pressure, you should be achieving good oxygenation. This can be a delicate balance, though. One must closely assess oxygenation in the delivery room. I've seen some proponents of using room air refuse to use any higher $\mathrm{F}_{\mathrm{IO}_{2}}$ while resuscitating a baby for longer than 3 minutes without there being an improvement in the child's condition. One has to be cognizant that ineffective prolonged use of room air could result in morbidity or mortality.

DiBlasi: You mentioned that babies don't really have a difficult time inhaling particles into their lungs: mainly meconium. In the premature population is there any interest in suctioning the oropharynx as the baby's head presents at the perineum, injecting surfactant into the airway prior to the first breath, so in the initial breath the surfactant would be distributed throughout the lung? Maybe we would not have to intubate as much? There are compelling animal data to support do- ing that, but where are we right now with humans?

Wiswell: Some small studies have addressed therapies like that, including obstetricians trying to do this in babies that are still inside the uterus. They tried to thread a catheter into the fetus's mouth or throat and then instill surfactant that way. A couple of small trials have tried instilling surfactant before the infant's first breath. Some people have tried to do it noninvasively, without intubating. However, there's not a lot of information yet. It's an intriguing approach, and you'd think you'd have more success doing that, unless the baby has taken some big breaths already.

Brown: You mentioned the SUPPORT trial, in which we were a primary center. You said that intubation surfactant wasn't found to be any better, but you could look at it the other way, which is that nasal CPAP was just as good. It's somewhat shocking, because isn't that what we were doing 30 years ago? Was that a practice at your institution? 
Wiswell: Good point. In my institution we've got some wonderful therapists who stay current with the medical literature and are interested in this. As part of a quality-improvement initiative we have been using early nasal CPAP, immediately after birth, even in the smallest of premature infants. And we've had promising results: our frequency of BPD is significantly lower. Essentially, though, we are comparing the two time periods before and after this approach, so it is not a randomized controlled trial. With this anecdotal experience we appear to be doing pretty well in my hospital, but what does that mean, without it being a randomized controlled trial? Not a lot.

Brown: The substudy from the SUPPORT trial was oxygen. In San Diego it definitely affected the management of oxygen saturation, and it changed our parameters when we saw the negative effect on mortality with lower saturation. We originally changed practice patterns without evidence, and later discovered we'd been doing harm, as with some other practices, such as steroids for babies. Are there plans for follow-up studies?

Wiswell: I think one or two others are looking at long-term outcomes in babies with lower saturation. You bring up a really good point, that everyone was expecting the opposite: that the lower-saturation group would do better, but that group had worse outcomes.

Brown: I thought it dovetailed well with your slides that showed that mortality has gone up and retinopathy of prematurity has gone down.

Wiswell: Yeah.

Cheifetz: I have not worked in a delivery room setting for many years, but I have heard my neonatal col- leagues debating room air versus $100 \%$ oxygen for neonatal resuscitation. From a distance, I have always wondered why it is either $21 \%$ or $100 \%$ oxygen? Why not $30 \%$ or $50 \%$ ? It was good to see the recommendation that blended oxygen is a recommended option. Is blended oxygen becoming the dominant approach?

Wiswell: I think most delivery rooms are starting at an $\mathrm{F}_{\mathrm{IO}_{2}}$ of 0.4 or 0.5 and titrating from there after seeing the response over several minutes and how the child looks clinically: if heart rate is up and they're doing well. In the delivery room I think it's good to have an oxygen saturation monitor placed soon after delivery, and most delivery rooms in the United States should be able to do that now. I think titrating oxygen is the best approach, and I doubt there's going to be any one $\mathrm{F}_{\mathrm{IO}_{2}}$ that's best in all circumstances. 